\title{
MULTIPLE POINTS AND WALLMAN COMPACTIFICATIONS
}

\author{
OLAV NJASTAD \\ (Received 4 February 1974) \\ Communicated by B. Mond
}

\begin{abstract}
Banaschewski (1963) and Frink (1964) generalized the compactification procedure of Wallman to obtain Hausdorff compactifications of Tychonoff spaces. Numerous papers have been devoted to the problem whether all Hausdorff compactifications may be obtained in this way, and for many classes of compactifications an affirmative answer has been given. This note is a contribution in this direction. We show that if a (Hausdorff) compactification $\alpha X$ of $X$ is the quotient space of a Wallman compactification $\gamma X$ in such a way that the set of multiple points of $\alpha X$ with respect to $\gamma X$ is not too large, then $\alpha X$ too is a Wallman compactification. The results are generalizations of earlier results of Steiner and Steiner (1968) and by the author (1966) for the special case that $\gamma X$ is the StoneCech-compactification.
\end{abstract}

\section{Preliminaries}

Let $\alpha X$ be an arbitrary (Hausdorff) compactification of the (Tychonoff) space $X$. We shall identify $X$ with a subset of $\alpha X$. Interior, closure, and boundary in $\alpha X$ are denoted by ${ }^{0}$, ${ }^{-}$, and $\partial$.

We start by presenting some general auxiliary results.

Lemma 1.1. If $G$ is open in $\alpha X$, then $G \subset(G \cap X)^{-0}$.

Proof. Let $x \in G$, and let $U$ be an arbitrary neighbourhood of $x$. Then $G \cap U$ is a neighbourhood of $x$, and hence contains a point $y \in X$, since $X$ is dense in $\alpha X$. Clearly $y \in G \cap X$, and so $x \in(G \cap X)^{-}$. This means that $G \subset(G \cap X)^{-}$, from which follows that $G \subset(G \cap X)^{-0}$, since $G$ is open.

COROLLARY 1.2. If $F$ is closed in $\alpha X$, then $\left(F^{0} \cap X\right)^{-0}=(F \cap X)^{-0}=F^{0}$.

Proof. Obviously $\left(F^{0} \cap X\right)^{-} \subset(F \cap X)^{-} \subset F$, hence $\left(F^{0} \cap X\right)^{-0} \subset$ $(F \cap X)^{-1} \subset F^{0}$. 
On the other hand, $F^{0} \subset\left(F^{0} \cap X\right)^{-0}$ by Lemma 1.1, from which the result follows.

COROLlaRY 1.3. If $F$ is closed in $\alpha X$, then $\partial\left[\left(F^{\circ} \cap X\right)^{-}\right] \subset \partial\left[(F \cap X)^{-}\right] \subset \partial F$

Proof. By Corollary 1.2 we have $\partial\left[\left(F^{0} \cap X\right)^{-}\right]=\left(F^{0} \cap X\right)^{-}-\left(F^{0} \cap X\right)^{-0}=$ $\left(F^{0} \cap X\right)^{-}-F^{0} \subset(F \cap X)^{-}-F^{0}=(F \cap X)^{-}-(F \cap X)^{-0}=\partial\left[(F \cap X)^{-}\right]$. Similarly we have $\partial\left[(F \cap X)^{-}\right]=(F \cap X)^{-}-F^{0} \subset F-F^{0}=\partial F$.

A base on a space shall always mean a base for the closed sets. A ring base is a base which is closed under finite unions and intersections.

Proposition 1.4. If $\mathscr{B}$ is a base on $\alpha X$, then $\mathscr{B}^{\prime}=[\mathscr{B} \cap X]^{-}$is also a base on $\alpha X$.

Proof. Let $H$ be closed in $\alpha X$ and $x \notin H$. Since $\alpha X$ is a normal space, there is a closed set $F$ such that $x \notin F, H \subset F^{\circ}$. Let $B \in \mathscr{B}$ be such that $F \subset B, x \notin B$. Then $x \notin(B \cap X)^{-}$, since $(B \cap X)^{-} \subset B$. According to Corollary 1.2 we have $(B \cap X)^{-} \supset B^{0}$, and $B^{0} \supset F^{0} \supset H$. Since $(B \cap X)^{-} \in \mathscr{B}^{\prime}$, this completes the proof.

(From Corollary 1.2 follows that if $F$ is regular, then $F=(F \cap X)^{-}$. So $\mathscr{B}^{\prime}=\mathscr{B}$ if $\mathscr{B}$ consists of regular sets.)

Now let $\gamma X$ be a compactification of $X$ such that $\gamma X \geqq \alpha X$, and let $\pi$ be the natural map of $\gamma X$ onto $\alpha X$. (That is: $\pi$ is a continuous, hence closed, map of $\gamma X$ onto $\alpha X$ which reduces to the identity on $X$.)

Proposition 1.5. Let $\mathscr{B}$ be a ring base on $\gamma X$. If $N$ is a neighbourhood of the closed set $F$ in $\alpha X$, then there is a set $B \in \mathscr{B}$ such that $F \subset \pi(B) \subset N$ (In particular $\pi(\mathscr{B})$ is a base on $\alpha X$.)

Proof. Let $N$ be an open neighbourhood of the closed set $F$ in $\alpha X$. Then $\pi^{-1}(F)$ and $\pi^{-1}(-N)$ are disjoint compact sets in $\gamma X$. Since the base $\mathscr{B}$ is a ring, there is a set $B \in \mathscr{B}$ such that $\pi^{-1}(F) \subset B, B \cap \pi^{-1}(-N)=\varnothing$. Clearly $F \subset \pi(B)$ and $\pi(B) \subset N$. By applying this result to the situation that $N=\alpha X-\{x\}$, where $x \notin F$, we conclude that $\pi(\mathscr{B})$ is a base.

COROLLARY 1.6. If $\mathscr{B}$ is a ring base on $\gamma X$, then $\mathscr{B}^{\prime}=[\mathscr{B} \cap X]^{-}$is a base on $\alpha X$.

Proof. Clearly $\mathscr{B} \cap X=\pi(\mathscr{B}) \cap X$, and so the result follows from Propotion 1.4 and Proposition 1.5.

\section{Multiple points and reduced bases}

In the following we assume that $\gamma X$ is a Wallman compactification, and wish to show that under certain conditions $\alpha X$ is a Wallman compactification too. It suffices to show that $\alpha X$ has a base $\mathscr{B}$ with the trace property with respect to $X$. (That is: If $\bigcap_{i=1}^{n} B_{i} \neq \varnothing$, then $\left(\bigcap_{i=1}^{n} B_{i}\right) \cap X \neq \varnothing$, for every finite col- 
lection $B_{1}, \cdots, B_{n} \in \mathscr{B}$.) Then the ring generated by $\mathscr{B} \cap X$ is a normal base on $X$ corresponding to $\alpha X$. See for example Steiner (1968) and Steiner and Steiner (1969). Furthermore we know that since $\gamma X$ is a Wallman compactification, $\gamma X$ has a ring base $\mathscr{W}$ with the trace property with respect to $X$ (f. ex. $\mathscr{W}=\mathrm{cl}_{\gamma X} \mathscr{L}$, where $\mathscr{L}$ is a normal base on $X$ corresponding to $\alpha X)$. See for example Steiner (1968). In the following we consider one fixed such base on $\gamma X$.

We define the reduced base $\mathscr{W}^{*}$ on $\alpha X$ corresponding to $\mathscr{W}$ by

$$
\mathscr{W}^{*}=\left\{Z^{-}: Z \in(\mathscr{W} \cap X) \& \pi^{-1}\left(Z^{-}\right) \subset B\right.
$$

for some $B \in \mathscr{W}$ for which $Z=B \cap X\}$.

Lemma 2.1. $\mathscr{W}^{*}$ has the trace property with respect to $X$.

Proof. Let $Z_{i}^{-} \in \mathscr{W}^{*}$ and let $B_{i} \in \mathscr{W}$ such that $Z_{i}=B_{i} \cap X, \pi^{-1}\left(Z_{i}^{-}\right) \subset B_{i}$, $i=1, \cdots, n$. Then $\bigcap_{i=1}^{n} \pi^{-1}\left(Z_{i}^{-}\right) \subset \bigcap_{i=1}^{n} B_{i}$, and $X \cap \bigcap_{i=1}^{n} B_{i}=\bigcap_{i=1}^{n} Z_{i}$ $=X \cap \bigcap_{i=1}^{n} Z_{i}^{-}$. Now assume that $X \cap \bigcap_{i=1}^{n} Z_{i}^{-}=\varnothing$. It follows that $\bigcap_{i=1}^{n} B_{i}=\varnothing$, since $\mathscr{W}$ has the trace property. Thus $\bigcap_{i=1}^{n} \pi^{-1}\left(Z_{i}^{-}\right)=\varnothing$, and hence $\bigcap_{i=1}^{n} Z_{i}^{-}=\varnothing$. This completes the proof.

We shall say that a point $x$ in $\alpha X$ is a multiple point with respect to $\gamma X$ if $\pi^{-1}(x)$ consists of more than one point. The set of such points will be denoted by $M_{\gamma x}$. (This generalizes the concept of multiple point in Njastad (1966), where $\gamma X$ is assumed to be the Stone-Čech-compactification.)

Proposition 2.2. Let $Z \in \mathscr{W} \cap X$, and assume that $\partial Z^{-} \cap M_{\gamma x}=\varnothing$. Then $Z^{-} \in \mathscr{W}^{*}$.

Proof. Let $B$ be an arbitrary set in $\mathscr{W}$ such that $B \cap X=Z$. By Lemma 1.1, $\pi^{-1}\left(Z^{-0}\right) \subset \operatorname{cl}_{\gamma X}\left(\pi^{-1}\left[Z^{-0}\right] \cap X\right)=\operatorname{cl}_{\gamma X}\left(Z^{-0} \cap X\right) \subset \operatorname{cl}_{\gamma X}\left(Z^{-} \cap X\right)=\operatorname{cl}_{\gamma X} Z \subset B$. Next let $x \in \pi^{-1}\left(\partial Z^{-}\right)$. We observe that $\pi(B) \supset Z^{-}$since $\pi$ is a closed mapping. So $\pi(x)$ is the image of some point $y$ in $B$. But $\pi(x) \notin M_{\gamma x}$ since $\partial Z^{-} \cap M_{\gamma x}=\varnothing$, hence $x$ must be equal to $y$. This means that $\pi^{-1}\left(\partial Z^{-}\right) \subset B$.

Thus we have shown that both $\pi^{-1}\left(\partial Z^{-}\right) \subset B$ and $\pi^{-1}\left(Z^{-0}\right) \subset B$, which means that $\pi^{-1}\left(Z^{-}\right) \subset B$. This completes the proof.

(We observe that $\pi^{-1}\left(Z^{-}\right) \subset B$ for every $B \in \mathscr{W}$ for which $B \cap X=Z$.)

\section{Zero-dimensional embedding of the multiple points}

Let $\mathscr{B}$ be a family of closed sets in a space $Y$. We shall say that a subset $A$ of $Y$ is $\mathscr{B}$-embedded ( $\mathscr{B}$-zero-dimensionally embedded) in $Y$ if $\mathscr{B}$ contains a base $\mathscr{B}_{0}$ for $Y$ such that $\partial B \subset Y-A$ for every $B \in \mathscr{B}_{0}$. Thus zero-dimensional embedding means $\mathscr{F}$-embedding, where $\mathscr{F}$ is taken to be the family of all closed sets. The condition of zero-dimensional embedding generally is weaker than that of $\mathscr{B}$-embedding. We notice that every subset of a $\mathscr{B}$-embedded set is $\mathscr{B}$-embedded. 
THEOREM 3.1. Let $\mathscr{W}$ be a ring base on $\gamma X$ with the trace property with respect to $X$. Assume that $M_{\gamma \alpha}$ is $\mathscr{B}$-embedded in $\alpha X$, where $\mathscr{B}$ is some (closed) family on $\alpha X$ such that $\mathscr{B} \cap X \subset \mathscr{W} \cap X$. Then $\mathscr{W}^{*}$ is a base on $\alpha X$, and $\alpha X$ is a Wallmann compactification.

Proof. Since $\mathscr{W}^{*}$ has the trace property with respect to $X$ (Lemma 2.1), it suffices to show that $\mathscr{W}^{*}$ is a base in order to know that $\alpha X$ is a Wallman compactification. By assumption $\mathscr{B}$ contains a base $\mathscr{B}_{0}$ such that $\partial B \subset \alpha X-M_{\gamma \alpha}$ for every $B \in \mathscr{R}_{0}$. For every such $B, B \cap X \in \mathscr{W} \cap X$, and $\partial\left[(B \cap X)^{-}\right] \subset \partial B$ according to Corollary 1.3. From Proposition 2.2 follows that $(B \cap X)^{-} \in \mathscr{W}^{*}$, and from Proposition 1.4 follows that $\left(\mathscr{B}_{0} \cap X\right)^{-}$is a base on $\alpha X$. Hence $\mathscr{W}^{*}$ is a base on $\alpha X$, which completes the proof.

Corollary 3.2. If $M_{\gamma \alpha}$ is $\pi(\mathscr{W})$-embedded in $\alpha X$, then $\alpha X$ is a Wallman compactification.

Proof. The result follows immediately from Theorem 3.1 and the observation that $\pi(\mathscr{W}) \cap X=\mathscr{W} \cap X$.

As usual, $\beta X$ shall denote the Stone-Čech-compactification of $X$.

PROPOSITION 3.3. If $X$ is a normal space and $M_{\beta x}$ is zero-dimensionally embedded in $\alpha X$, then $\alpha X$ is a Wallman compactification.

Proof. Let $\mathscr{W}$ denote the family of all sets of the form $\mathrm{cl}_{\beta X} C, C$ closed in $X$. Then $\mathscr{W} \cap X$ consists of all closed sets in $X . \mathscr{W}$ is a base on $\beta X$ and has the trace property with respect to $X$, since $X$ is normal. Let $\mathscr{B}$ denote the family of all closed sets in $\alpha X$. By assuption $M_{\beta \alpha}$ is $\mathscr{B}$-embedded in $\alpha X$, and $\mathscr{B} \cap X=\mathscr{W} \cap X$. So the result follows by Theorem 3.1.

We recall that two sets in $\alpha X$ are said to be far (with respect to the corresponding proximity) if their closures in $\alpha X$ are disjoint. We shall say that a set $A$ is weakly p-embedded in $\alpha X$ if every two disjoint (open-closed) sets in a partition of $A$ are far. Clearly every closed set (and every connected set) is weakly $p$-embedded.

LEMMA. 3.4. Let $\mathscr{B}$ be a base on $\alpha X$ such that if $N$ is a neighbourhood of a closed set $C$ then there is a set $B \in \mathscr{B}$ such that $C \subset B \subset N$. If a set $\Omega$ is weakly p-embedded in $\alpha X$, then $\Omega$ is $\mathscr{B}$-embedded in $\alpha X$.

Proof. Let $A$ be a closed set in $\alpha X$ and let $x \notin A$. Then there is a closed set $F$ such that $A \cap F^{0}, x \notin F$, and by the zero-dimensional embedding there is a closed set $H$ such that $F \subset H^{0}, x \notin H, \partial H \cap \Omega=\varnothing$. Now consider the sets $B=\Omega \cap H, C=\Omega-H . B$ and $C$ are closed in $\Omega$, hence $B^{-} \cap C^{-}=\varnothing$ by the weak $p$-embedding. We have $(x \cup C)^{-} \cap(A \cup B)^{-}=\left(x \cup C^{-}\right) \cap\left(B^{-} \cup A\right)=$ $\left(x \cap B^{-}\right) \cup\left(C^{-} \cap B^{-}\right) \cup(x \cap A) \cup\left(C^{-} \cap A\right)$. Obviously $x \cap B^{-}=\varnothing, x \cap A$ $=\varnothing$. Thus $(A \cup B)^{-} \subset \alpha X-\left[(x \cup C)^{-}\right]$, and so there is by assumption (and the normality of $\alpha X)$ a set $K$ in $\mathscr{B}$ such that $(A \cup B)^{-} \subset K^{0}, K \subset \alpha X-\left[(x \cup C)^{-}\right]$. 
It follows that $K^{0} \cap \Omega \supset(A \cup B) \cap \Omega=B,(\alpha X-K) \cap \Omega \supset\left(x \cup C^{-}\right) \cap \Omega \supset C$. This means that $\partial K \cap \Omega=\varnothing$. Since $A \subset K, x \notin K$, this shows that $\Omega$ is $\mathscr{B}$-embedded in $\alpha X$, which was to be proved.

THEOREM 3.5. If $\gamma X$ is a Wallman compactification and $M_{\gamma \alpha}$ is contained in a weakly p-embedded and zero-dimensionally embedded subset $\Omega$ of $\alpha X$, then $\alpha X$ is a Wallman compactification.

Proof. According to Lemma 3.4 and Proposition 1.5, $\Omega$ and hence $M_{\gamma^{\alpha}}$ is $\pi(\mathscr{W})$-embedded in $\alpha X$, where $\mathscr{W}$ is a ring base on $\gamma X$ wiht the trace property with respect to $X$. So the result follows by Corollary 3.2 .

COROLLARY 3.6. If $\gamma X$ is a Wallman compactification, and $M_{\gamma \alpha}$ is contained in a set $\Omega$ which is normally and zero-dimensionally embedded in $\alpha X$, then $\alpha X$ is a Wallman compactification.

Proof. Let $A$ and $B$ be disjoint open-closed sets in $\Omega$. The function $f$ which is 1 on $A, 0$ on $\Omega-A$, is continuous on $\Omega$. The normal embedding of $\Omega$ implies that $f$ has a continuous extension to $\alpha X$. From this follows that $A^{-} \cap B^{-}=\varnothing$. Thus $\Omega$ is weakly $p$-embedded in $\alpha X$, and the result follows from Theorem 3.5. (This result is in Njastad (1966) for the case that $\gamma X=\beta X$.)

\section{Countable sets of multiple points}

A family $\mathscr{I}$ of continuous functions on a set $Y$ is said to be separating if for every closed set $F$ and point $x$ not in $F$ there is a function $f \in \mathscr{I}$ such that $f(F)=\{0\}, f(x)=1, f(Y) \subset[0,1]$. In particular $\mathscr{C}(\alpha X)$ is a separating family on $\alpha X$. For every real number $r$ we write $Z_{r}(f)=\{z: f(z) \leqq r\}$, and we denote by $\mathscr{F}[\mathscr{I}]$ the family of all sets $Z_{r}(f), f \in \mathscr{I}$. We denote by $\mathscr{E}(X, \alpha X)$ the family of all functions on $X$ which are continuously extendable to $\alpha X$. It is easily seen that $\mathscr{F}[\mathscr{E}(X, \alpha X)]=\mathscr{F}[\mathscr{C}(\alpha X) \cap X$.

LEMMA 4.1. A countable set $M$ in a space $Y$ is $\mathscr{F}[\mathscr{J}]$-embedded for every separating family $\mathscr{I}$.

Proof. Let $F$ be closed in $Y, x \notin F$, and let $f$ be a function in $\mathscr{I}$ such that $f(x)=1, f(F)=\{0\}, f(Y) \subset[0,1]$. We observe that $\partial Z_{r}(f) \subset\{z: f(z)=r\}$, hence the sets $\partial Z_{r}(f)$ are disjoint. So $M \cap \partial Z_{r}(f)=\varnothing$ for at least one $r$, since $M$ is countable. This shows that $M$ is $\mathscr{F}[\mathscr{F}]$-embedded in $Y$, which was to be proved.

The spaces which admit separating families are the completely regular ones, so in particular we conclude that every countable set in $\alpha X$ is zero-dimensionally embedded.

PRoposition 4.2. Let $\mathscr{W}$ be a ring base on $\gamma X$ with the trace property with respect to $X$, and assume that there is a separating family $\mathscr{I}$ on $\alpha X$ such that 
$\mathscr{F}[\mathscr{F}] \cap X \subset \mathscr{W} \cap X$. Then $\alpha X$ is a Wallman compactification if $M_{\gamma \alpha}$ is countable.

Proof. The result follows from Theorem 3.1, since by Lemma 4.1, $M_{\gamma \alpha}$ is $\mathscr{F}[\mathscr{I}]$-embedded.

Proposition 4.3. If $\gamma X$ is a Wallman compactification, and $M_{\gamma \alpha}$ is countable and weakly p-embedded in $\alpha X$, then $\alpha X$ is a Wallman compactification.

Proof. From Lemma 4.1 follows that $M_{\gamma x}$ is zero-dimensionally embedded in $\alpha X$, and so the result follows by Theorem 3.5.

COROLlary 4.4. If $\gamma X$ is a Wallman compactification and $M_{\gamma \alpha}$ is countable and closed (in particular if $M_{\gamma \alpha}$ is finite), then $\alpha X$ is a Wallman compactification.

Proof. Every closed set is weakly $p$-embedded, and Proposition 4.3 applies.

PROPOSITION 4.5. Let $\mathscr{W}$ be a ring base on $\gamma X$ with the trace property with respect to $X$, and assume that $\mathscr{F}[\mathscr{E}(X, \alpha X)] \subset \mathscr{W} \cap X$. Then $\alpha X$ is a Wallman compactification if $M_{\gamma \alpha}$ is countable.

Proof. $M_{\gamma \alpha}$ is $\mathscr{F}[\mathscr{C}(\alpha X)]$-embedded in $\alpha X$ by Lemma 4.1 , and so the result follows by Theorem 3.1 and fact that $\mathscr{F}[\mathscr{C}(\alpha X)] \cap X=\mathscr{F}[\mathscr{E}(X, \alpha X)]$.

COROLlary 4.6. Assume that $\mathscr{W}$ is a ring base on $\gamma X$ with the trace property with respect to $X$, and that $\mathscr{W} \cap X \supset \mathscr{F}[\mathscr{E}(X, \gamma X)]$. Then $\alpha X$ is a Wallman compactification if $M_{\gamma \alpha}$ is countable.

Proof. The result immediately follows from Proposition 4.5 , since $\mathscr{F}[\mathscr{E}(X, \alpha X)] \subset \mathscr{F}[\mathscr{E}(X, \gamma X)]$.

We remark that if $\gamma X=\beta X$, then by choosing

$$
\mathscr{W}=\mathrm{cl}_{\beta X} \mathscr{F}[\mathscr{C}(X)]=\mathrm{cl}_{\beta X} \mathscr{F}[\mathscr{E}(X, \beta X)]
$$

we have $\mathscr{W} \cap X=\mathscr{F}[\mathscr{E}(X, \beta X)]$. Hence it follows that if $M_{\beta \alpha}$ is a countable set, then $\alpha X$ is a Wallman compactification. (This is the result of Steiner and Steiner (1969)).

\section{Conclusion}

Important classes of compact spaces which are Wallman compactifications (of their dense subspaces) are the compact metric spaces (see, for example, Aarts (1968), Steiner and Steiner (1969)) and the compact linearly ordered spaces (see, for example, Biles (1970), Hamburger (1971)). Let $T$ be such a compact space, and $X$ a dense subspace. Then the compactification $\gamma X=T$ is usually not the Stone-Cech-compactification of $X$. Let $\alpha X$ be a compactification of $X$ such that $\alpha X \leqq \gamma X$. Then $M_{\gamma \alpha}$ may be sufficiently small (with respect to the con- 
ditions of the theorems in this note) to insure that $\alpha X$ is a Wallman compactification, while $M_{\beta \alpha}$ may be rather large. Thus in some situations it is possible to conclude from properties of $M_{\gamma \alpha}$ that $\alpha X$ is a Wallman compactiffication, while the same conclusion may not be obtained by considering $M_{\beta \alpha}$. Simple examples are obtained for example by identifying a finite number of points in a closed subset of a Euclidean space (the closed set being a compactification of some dense subset not containing the identified points).

\section{References}

J. M. Aarts (1968), Every metric compactification is a Wallman compactificotion, Proc. Int. Symp. on Topology and its Applications (Herceg-Novi (1968)).

B. Banaschewski (1963), 'On Wallman's method of compactification', Math. Nachr. 27, 105-140.

C. M. Biles (1970), 'Wallman-type compactifications', Proc. Amer. Math. Soc. 25, 363-368.

O. Frink (1964), 'Compactifications and semi-normal spaces', Amer.J. Math. 86, 602-707.

P. Hamburger (1971), 'On Wallman-type, regular Wallman-type and Z-compactifications', Period. Math. Hungar. 1, 303-309.

O. Njastad (1966), 'On Wallman-type compactifications', Math. Z. 91, 267-276.

E. F. Steiner (1968), 'Wallman spaces and compactifications', Fund. Math. 61, 295-304.

A. K. Steiner and E. F. Steiner (1968), 'Products of compact metric spaces are regular Wallman', Indag. Math. 30, 426-430.

A. K. Steiner and E. F. Steiner (1969), 'On countable multiple point compactifications', Fund. Math. 65, 133-137.

University of Colorado

Boulder

U.S.A.

Current Address:

Technical University of Norway

7000 Trondheim

Norway. 